\title{
Overshooting and semiconvection: structural changes and asteroseismic signatures
}

\author{
A. Noels · J. Montalban • A. Miglio • M. Godart • \\ P. Ventura
}

Received: 14 September 2009 / Accepted: 9 November 2009 / Published online: 27 November 2009

(C) Springer Science+Business Media B.V. 2009

\begin{abstract}
Overshooting and semiconvection are two poorly known mechanisms which affect the extent and the efficiency of chemical mixing outside classical convection zones in stars. We discuss the uncertainties and the inferences of those processes in main sequence stars burning hydrogen in a convective core. We then focus on the asteroseismic signatures of partially or fully mixed zones surrounding the convective core, through the detailed shape of the induced chemical composition profile. We emphasize the potential power of asteroseismology to determine the internal structure of stars and thus to help us understand the physical processes at work inside the stars.
\end{abstract}

Keywords Stellar structure · Overshooting •

Semiconvection · Asteroseismology

\section{Introduction}

Apart from low mass stars $\left(M \lesssim 1.1 M_{\odot}\right)$, main sequence stars burn hydrogen inside a convective core where matter is fully mixed. This leads to a mean molecular weight constant throughout the core and increasing with time as hydrogen is transformed into helium. In between this core and the homogeneous envelope, a region of varying mean molecular weight ( $\mu$-gradient region) builds up, whose detailed shape is of crucial importance for asteroseismic studies. It induces

A. Noels $(\bowtie) \cdot$ J. Montalban · A. Miglio $\cdot$ M. Godart Institut d'Astrophysique et de Géophysique, University of Liège, Liège, Belgium

e-mail: Arlette.Noels@ulg.ac.be

P. Ventura

Osservatorio Astronomico di Roma, Monte Porzio, Italy a growing peak in the Brünt-Väisälä frequency and permits the appearance of what are called mixed modes whose detection bears in turn the signature of the structure of these layers. Some physical processes may alter the shape of the $\mu$-gradient, mostly through a full or partial mixing of some radiatively stable layers above the limit of the convective core. This is the case for rotation, overshooting, semiconvection, and diffusion. We shall here mainly focus on the mixing induced by overshooting, and we shall briefly address the problem of semiconvection in small mass main sequence stars.

\section{Overshooting}

The problem of overshooting can be very simply stated. The limit of the convective core is fixed by the Schwarzschild criterion, which imposes the equality of the radiative and the adiabatic temperature gradients $\left(\nabla_{\mathrm{rad}}=\nabla_{\mathrm{ad}}\right)$. However, a convective bubble crossing the edge of the core has an acceleration whose sign is determined by the Ledoux criterion. It is thus decelerated in the radiatively stable region, but its velocity remains different from zero. The result is a region contaminated by wandering bubbles surrounding the convective core.

\subsection{Extent of the overshooting region}

In the classical treatment of overshooting, the distance $d_{\mathrm{ov}}$ covered by the convective eddies outside the convective core is equal to a fraction $\alpha_{\mathrm{ov}}$ of the pressure scale height $H_{\mathrm{P}}$ except for very small convective cores, smaller than $H_{\mathrm{P}}$ estimated at the core boundary $r_{\mathrm{c}}$. This is written as

$d_{\mathrm{ov}}=\alpha_{\mathrm{ov}} \min \left(r_{\mathrm{c}}, H_{\mathrm{P}}\right)$ 
Estimates of the overshooting parameter $\alpha_{\mathrm{ov}}$ have been made through fittings of theoretical models with binaries (see for instance Andersen et al. 1990; Ribas and Jordi 2000; Claret 2007) or with stellar clusters (see for instance Bertelli et al. 1986; Chiosi et al. 1989; Maeder and Meynet 1991; Stothers and Chin 1992; Demarque et al. 1994). In most of these analyses, the overshooting parameter is found to be different from zero and smaller than 0.5 , the favored value being around 0.2. Moreover, $\alpha_{\mathrm{ov}}$ seems to slightly increase with the stellar mass.

On the theoretical side, different attempts have been made to derive the dimension of the overshooting region (see for instance Bressan et al. 1981). Roxburgh (1978, 1989) proposed a global condition, in the frame of the mixing length theory, to describe the whole region $\left[r_{1}, r_{2}\right]$ affected by convection, i.e., the convective core and overshooting region, written as

$\int_{r_{1}}^{r_{2}}\left(L_{\mathrm{rad}}-L\right) \frac{1}{T^{2}} \frac{\mathrm{d} T}{\mathrm{~d} r} \mathrm{~d} r=0$

Since the temperature $T$ decreases monotonically with $r$, this relation demonstrates that in some layers the energy carried by radiation is smaller than the total energy $\left(L_{\mathrm{rad}}<L\right)$, which is the case inside the purely convective core, while some layers must have $L_{\text {rad }}>L$, thus defining the overshooting region. Zahn (1991) confirmed the validity of this integral constraint in the case of a convective core where convection is adiabatic but questioned its use for a convective envelope where departures from adiabaticity are large. His recommendations to fix the extent of the overshooting region are to use (1) a significant fraction of the core radius for a convective core and (2) a pressure scale height for a convective envelope.

\subsection{Chemical profile in the overshooting region}

The efficiency of the mixing in the overshooting region is very difficult to assess. Most evolution codes such as ASTEC (Christensen-Dalsgaard 2008), FRANEC (Degl'Innocenti et al. 2008), YREC (Demarque et al. 2008), Geneva code (Eggenberger et al. 2008), TGEC (Hui-BonHua 2008), CESAM (Morel and Lebreton 2008), STAROX (Roxburgh 2008), and CLES (Scuflaire et al. 2008) choose an instantaneous mixing, meaning that the fully mixed region extends over the convective region and the overshooting region. The $\mu$-gradient region then only starts at the external boundary of the overshooting region.

Mixing can also be treated as a diffusion process. In the ATON (Ventura et al. 1998, 2008) implementation, noninstantaneous mixing takes place in the convective core and in the overshooting region. Outside the convective region, turbulent velocities are assumed to decay exponentially as $u=u_{\mathrm{b}} \exp -\left(\frac{1}{\zeta f_{\text {thick }}} \ln \frac{P}{P_{\mathrm{b}}}\right)$

where $u_{\mathrm{b}}$ and $P_{\mathrm{b}}$ are the velocity (estimated in the framework of the adopted convection theory) and the pressure at the convective boundary, and $f_{\text {thick }}$ is the thickness of the convective region in terms of $H_{\mathrm{P}}$. The diffusion coefficient is then written as

$D=\frac{u \beta H_{\mathrm{P}}}{3}$

where $\beta$ is adapted to ensure the continuity of the diffusion coefficient at the core boundary. In order to mimic the main sequence width obtained with an instantaneous mixing parameterized with $\alpha_{\mathrm{ov}}=0.2, \zeta$ must be of the order of 0.02 .

A similar approach can be found in GARSTEC (Weiss and Schlattl 2008). The diffusion coefficient also has an exponentially decreasing behavior written as

$D(z)=D_{0} \exp \frac{-2 z}{f H_{\mathrm{P}}}$

where $f$ is a parameter whose standard value is $0.016, z$ is the distance from the core boundary, and $D_{0}$ is computed from the mixing length theory (MLT) convective velocity. Instead of a sharp-edged hydrogen profile found with no overshooting or with an instantaneous mixing, a diffusive approach leads to a smoother profile.

\subsection{Temperature gradient in the overshooting region}

The overshooting region is radiatively stable, which implies a temperature gradient, $\nabla$, smaller than the adiabatic temperature gradient, $\nabla_{\mathrm{ad}}$. Since the temperature difference between the overshooting eddies and the surrounding medium is proportional to $\left(\nabla-\nabla_{\mathrm{ad}}\right)$, a cooling ensues and, as a result, $\nabla$ is greater than the value which would occur without overshooting, i.e., $\nabla_{\text {rad }}$, and

$\nabla_{\mathrm{rad}} \lesssim \nabla \lesssim \nabla_{\mathrm{ad}}$

According to a terminology proposed by Zahn (1991), in the case of a very efficient convective heat transport in the stable layers penetrated by turbulent eddies, mainly outside convective cores, the temperature gradient is close to the adiabatic one and the process may be described as a penetrative convection. When the efficiency is low, eddies crossing the edge of a convective region, mainly a convective envelope, can transport chemicals and mix the stable layers without altering the radiative temperature gradient. The term overshooting applies to this inefficient penetration. 
Figure 1 (upper panel) shows the slightly different behavior in the HR diagram of a $10 M_{\odot}$ evolving under the assumption of either overshooting $\left(\nabla=\nabla_{\text {rad }}\right.$, full line) or penetrative convection $\left(\nabla=\nabla_{\mathrm{ad}}\right.$, dotted line) with an extramixed region extending over $0.2 H_{\mathrm{P}}$. A small adjustment of $\alpha_{\mathrm{ov}}$ from 0.2 to 0.175 in the overshooting assumption leads to exactly the same extra-mixed zone, although the extension of the convective core remains slightly larger in the overshooting case. With this adjustment of $\alpha_{\mathrm{ov}}$, the BruntVäisälä frequency defined as

$N^{2}=\frac{g}{r} \frac{\mathrm{d} \ln P}{\mathrm{~d} \ln r}\left[\left(\frac{\partial \ln \varrho}{\partial \ln T}\right)_{\mathrm{P}, \mu}\left(\nabla_{\mathrm{ad}}-\nabla\right)-\nabla_{\mu}\left(\frac{\partial \ln \rho}{\partial \ln \mu}\right)_{\mathrm{T}, \mathrm{P}}\right]$

where $g$ is the local gravity and $\nabla_{\mu}$ is $\mathrm{d} \ln \mu / \mathrm{d} \ln P$, only shows a slight difference in the extra-mixed region (Fig. 1,

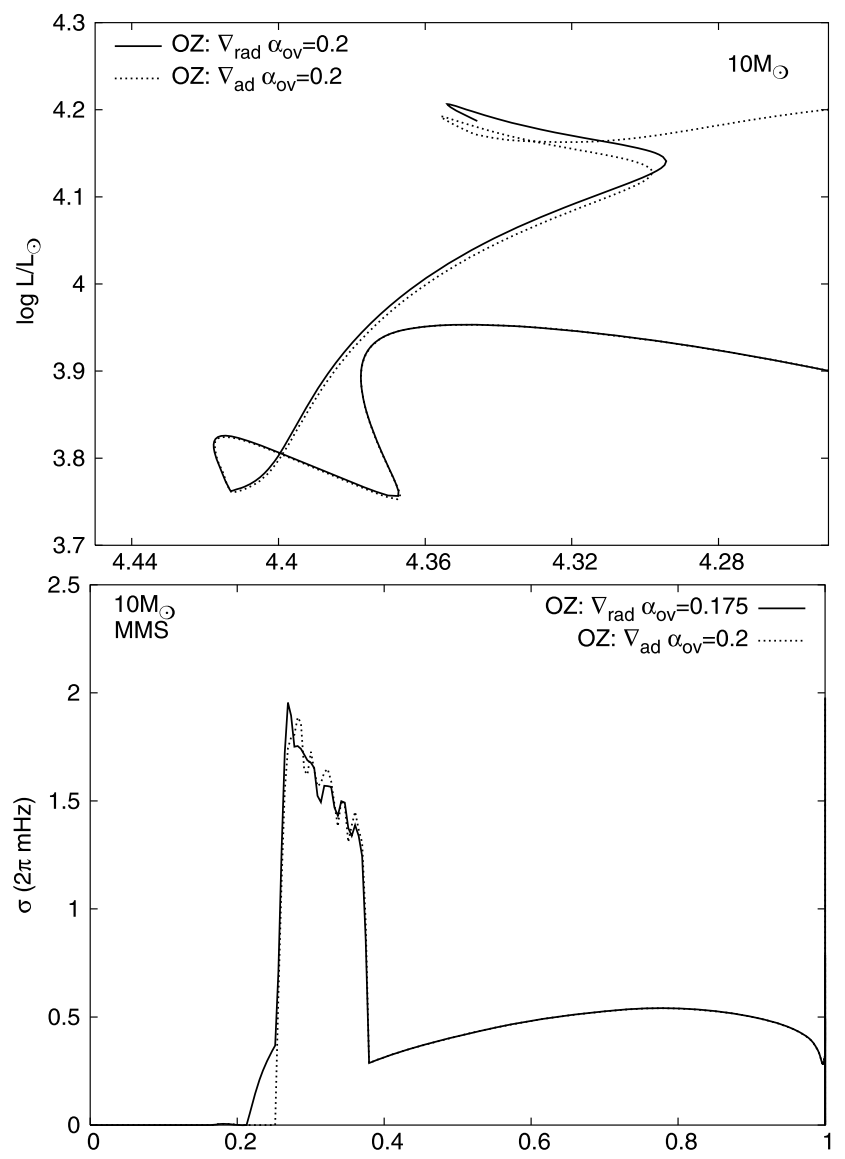

Fig. 1 Upper panel: HR evolutionary tracks of a $10 M_{\odot}$ star computed with different assumptions for the temperature gradient in the extra-mixed zone $\left(\alpha_{\mathrm{ov}}=0.2\right)$ surrounding the convective core. Full line corresponds to overshooting $\left(\nabla=\nabla_{\text {rad }}\right)$, while dotted line indicates penetrative convection $\left(\nabla=\nabla_{\mathrm{ad}}\right)$. Lower panel: Same for the Brunt-Väisälä frequency in a model with a central hydrogen content of 0.31 . The value of $\alpha_{\mathrm{ov}}=0.2$ in the overshooting assumption has been changed from 0.2 to 0.175 to reproduce the same overall mixed region in both cases (Godart 2006) lower panel) (Godart 2006). This difference is however high enough to affect g-mode and low-order p-mode frequencies at the level of precision of current asteroseismic observations (Godart 2007). A detection of such a small effect however requires a prior knowledge of the stellar global parameters as well as a reliable mode identification.

\section{Semiconvection}

For stars in the mass range $1.6 M_{\odot} \lesssim M \lesssim 30 M_{\odot}$ and a solar metallicity, the convective core mass decreases as hydrogen is transformed into helium due to the opacity decrease. A hydrogen profile is progressively built as this withdrawal takes place. On the contrary, in the mass range $1.1 M_{\odot} \lesssim M \lesssim 1.6 M_{\odot}$, the convective core increases in mass at least during part of the main sequence phase. Figure 2 illustrates this behavior of the convective core mass given as a function of the central hydrogen abundance (left panel). This is due to the fact that the relative importance of the pp chain decreases as the temperature increases while the $\mathrm{CNO}$ cycle contributes more and more to the energy generation; the larger temperature sensitivity of the CNO cycle favors the presence and the growing of a convective core. A somewhat similar situation occurs in massive stars which undergo a growing importance of the radiation pressure with a consecutive decrease in $\nabla_{\mathrm{ad}}$. In both cases, this generates a discontinuity in the hydrogen profile but also in $\nabla_{\mathrm{rad}}$ at the convective core boundary, leading to the well-known problem of semiconvection. The canonical treatment of semiconvection consists in assuming a partial chemical mix-

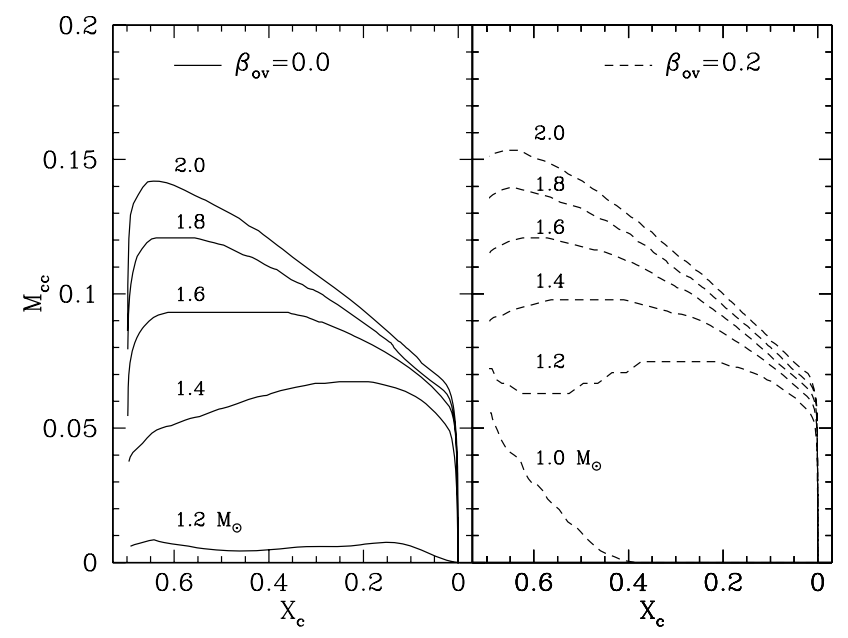

Fig. 2 Fractional mass of the convective core as a function of the central hydrogen abundance for main sequence stars in the mass range [1.0 $\left.M_{\odot}, 2.0 M_{\odot}\right]$. In the left panel, models have been computed with no overshooting; in the right panel, an overshooting parameter of 0.2 $\left(\alpha_{\text {ov }}\right.$ in (1)) was assumed (Miglio et al. 2008) 
ing in order to obtain a neutrality condition with Schwarzschild's or Ledoux's criterion (Stothers and Chin 1976; Chiosi and Maeder 1986).

Such a partial mixing can be achieved assuming various scenarios (Popielski and Dziembowski 2005). In the approach developed by Langer et al. (2008), semiconvection is treated as a diffusive process with a diffusion coefficient $D_{\mathrm{sc}}$ written as

$D_{\mathrm{sc}}=\alpha \frac{\kappa_{\mathrm{r}}}{6 c_{\mathrm{P}} \varrho} \frac{\nabla-\nabla_{\mathrm{ad}}}{\nabla_{\mathrm{L}}-\nabla}$

where $\alpha$ is an efficiency factor of the order of $0.1, c_{\mathrm{P}}$ is the specific heat at constant pressure, $\kappa_{\mathrm{r}}$, the radiative conductivity, $\varrho$, the density, and $\nabla_{\mathrm{L}}$, the Ledoux gradient $\left(=\nabla_{\mathrm{ad}}+\frac{\beta}{4-3 \beta} \nabla_{\mu}, \beta\right.$ is the ratio of gas pressure to total pressure) (see also Grossman and Taam 1996). The underlying idea is that semiconvective regions generate vibrationally unstable $g^{+}$-modes which drive a partial mixing (Kato 1966; Gabriel and Noels 1977). The chemical profile resulting from such a mixing washes out the discontinuity, leaving a much smoother profile (Silva-Aguirre et al. 2009) of the same overall aspect as a diffusive overshooting profile (see Sect. 2.2).

\section{Structural changes induced by overshooting}

The most striking effect of overshooting on the global properties of stars is a widening of the main sequence in the HR diagram. This means that, at a given effective temperature, a star evolving with overshooting is less evolved than its counterpart computed with no extra-mixed region. Overshooting also increases the duration of that phase by a significant amount depending on the value of $\alpha_{\mathrm{ov}}$.

As to the detailed internal stellar structure, overshooting increases the mass of the "classical" convective core in small mass stars. This is due to the fact that an extra-mixing brings fresh ${ }^{3} \mathrm{He}$ within the convective core, which prevents ${ }^{3} \mathrm{He}$ from reaching or coming close to its equilibrium value. The temperature sensitivity of the pp chain is then enhanced, which favors convection. Figure 2 shows the different behavior with central hydrogen abundance of models computed without and with overshooting $\left(\alpha_{\mathrm{ov}}=0.2\right.$, see (1)) in this particular mass range (Miglio et al. 2008). One can see that overshooting reduces the minimum mass of stars having a convective core during at least part of the main sequence, but it also decreases the maximum mass of stars having a growing convective core during that phase. This means that the conditions for semiconvection become more and more difficult to fulfill as $\alpha_{\mathrm{ov}}$ increases; overshooting essentially washes out semiconvection.

\section{Asteroseismic signatures}

\subsection{Occurrence of mixed modes}

At zero age main sequence, the p-mode frequency spectrum is clearly separated from the g-mode spectrum. As the evolution proceeds, a $\mu$-gradient develops outside the convective core and a growing peak appears in the BruntVäisälä frequency. This means that g-mode frequencies increase and become comparable to those of low order pmodes. Both frequency spectra interact without actually crossing. This is known as the avoided crossing phenomenon (Aizenman et al. 1977). Mixed modes having a gmode behavior close to the center and a p-mode behavior in the external layers are the clear signature of the time evolution of the $\mu$-gradient. At a given effective temperature, a model computed with overshooting is less evolved (see Sect. 4), so the avoided crossing phenomenon is less pronounced. As a result, fewer mixed modes show up in the spectrum of a star computed with overshooting. Thorough analyses of this phenomenon have been performed with models of $\delta$ Scuti stars clearly demonstrating the presence of an asteroseismic signature of overshooting in the mixed mode properties of the pulsation spectrum (see for instance Dziembowski and Pamyatnykh 1991; Goupil et al. 1993; Audard et al. 1995).

\subsection{Frequency separations}

The avoided crossing phenomenon also has a direct effect on the frequency separations which, because of the presence of mixed modes, no longer satisfy the asymptotic regularities in the spacings. The ratio of the two frequency separations

$\frac{d_{k, 0}^{(1)}}{d_{k, 0}^{(2)}}=\frac{v_{k, 0}+v_{k-1,0}-2 v_{k-1,1}}{v_{k, 0}-v_{k-1,2}}$

where $v_{k, l}$ is the frequency of the mode defined by its order $k$ and its degree $l$, and its variation with frequency, are indeed very sensitive to the internal structure, as was shown by Audard et al. (1995) in $\delta$ Scuti models of $2 M_{\odot}$ computed with or without overshooting. While radial models penetrate down to the center, modes of degree $l$ are reflected either by the Lamb frequency of same degree $\left(S_{\mathrm{L}}=\sqrt{l(l+1) c^{2} / r^{2}}\right)$ or the Brunt-Väisälä frequency (7), depending on which is the largest at the mode frequency. An $l=1$ mode will thus be more sensitive to the increase in $N^{2}$ since it travels deeper into the star. As the evolution proceeds, higher and higher order modes are affected. As a result, $d_{k, 0}^{1}$ is more sensitive to evolutionary effects than $d_{k, 0}^{2}$, which explains the behavior of this ratio (see also Sect. 6.2). 


\subsection{Period spacing in high order g-modes}

High order g-modes have been detected in $\gamma$ Doradus and SPB stars. According to the asymptotic theory, periods of high order g-modes are regularly spaced with a spacing $\left(\Delta P=P_{k+1}-P_{k}\right)$ decreasing as the degree of the modes increases; here the period spacing plays the role of the frequency spacing found for acoustic modes. As a star evolves along the main sequence, the higher and higher peak in the Brunt-Väisälä frequency (see Sect. 5.1) affects the regularity in $\Delta P$. This problem was first addressed in the case of white dwarfs (Brassard et al. 1992; Montgomery et al. 2003). Turning to $\gamma$ Doradus and slowly pulsating B (SPB) stars, Miglio et al. (2008) showed that $a$ sharp feature in the Brunt-Väisälä frequency shows up as a periodic component in the g-mode period spacing. The period of this component is written in terms of the radial order $k$,

$\Delta k \simeq \frac{\Pi_{\mu}}{\Pi_{0}}$

where $\Pi_{x}$ is the buoyancy radius defined as

$\Pi_{x}^{-1}=\int_{x_{0}}^{x} \frac{|N|}{x^{\prime}} \mathrm{d} x^{\prime}$

In these relations, $x$ stands for the fractional radius with a subscript 0 for the convective core boundary and a subscript $\mu$ for the location of the sharp feature in $N$, i.e., the external limit of the $\mu$-gradient region. One can see from (10) and (11) that the period in $\Delta P$ decreases as the extent of the $\mu$ gradient region $\left[x_{0}, x_{\mu}\right]$ increases, i.e., as the evolution proceeds and that, in the limiting case of a discontinuity at the boundary of the convective core, an infinite value for $\Delta k$ is obtained. This is illustrated in Fig. 3, where three evolutionary states in the evolution of a $6 M_{\odot}$ star are considered. In the top panel the Brunt-Väisälä frequency $N$ and the hydrogen content $X$ are shown. The sharp feature in $N$ moves out as the star evolves, i.e., as the convective core shrinks. This translates into a periodicity in $\Delta P$ (bottom panel) which decreases from the zero age main sequence (ZAMS) to the terminal age main sequence (TAMS) (Miglio et al. 2008).

Instantaneous overshooting extends the fully mixed region, which means that the $\mu$-gradient region is shifted towards layers more distant from the center. In stars massive enough $\left(M \gtrsim 2 M_{\odot}\right)$ to have a well-developed convective core with no nuclear reactions outside its boundary, the shift leaves a $\mu$-gradient region parallel to what is found with no overshooting and whose extent in buoyancy radius is the same in both cases. Since $N$ is indeed very small in the layers located outside the $\mu$-gradient region, the period $\Delta k$ is related to the extent and not to the location of the $\mu$-gradient region, and no changes are expected in $\Delta k$ for a similar central value of $X$. This $X$ value is however reached at a different effective temperature since the evolution with overshooting is slower. The situation is very different in smaller mass stars, where nuclear reactions also take place outside the convective core and affect the hydrogen profile. The $\mu$ gradient region is shifted, but its exact shape is different with or without overshooting. This is illustrated in Fig. 4 (Miglio et al. 2008). The difference in $\Delta P$ is even larger for lower masses such as $1.2 M_{\odot}$ for example, since a model without overshooting does not keep a convective core during main sequence evolution.

When a diffusive overshooting is adopted, the $\mu$-gradient is smoother and so is the Brunt-Väisälä frequency behavior. This would also be the case with a rotational mixing, which can be closely mimicked by introducing a turbulent diffusion coefficient into the diffusion equation. With a smoother sharp feature in $N$, the amplitude of the oscillation in the period spacing is modulated by a factor $1 / P_{k}$. This modulation clearly shows up in Fig. 5, which is similar to Fig. 3 for models computed with a turbulent diffusion $D_{\mathrm{T}}=5000 \mathrm{~cm} \mathrm{~s}^{-2}$ (Miglio et al. 2008, 2009). The modulation is so strong that the oscillation nearly vanishes.

The difference between instantaneous overshooting (sharply defined $\mu$-gradient) and diffusive overshooting or rotational mixing (smoother $\mu$-gradient) can also be directly seen in the frequencies of low order g-modes. Figure 6 shows the frequencies of $l=0-3$ modes as a function of the effective temperature for main sequence $10 M_{\odot}$ models computed with an instantaneous overshooting parameter of 0.1 (gray dots) compared to models computed with a turbulent diffusion coefficient $D_{\mathrm{T}}=7 \times 10^{4} \mathrm{~cm} \mathrm{~s}^{-2}$ (open circles). The differences can be as large as $5 \mu \mathrm{Hz}$, of the order of or even larger than the rotational splitting derived with a rotational velocity of about $50 \mathrm{~km} \mathrm{~s}^{-1}$ typical of a $\beta$ Cephei star (Montalbán et al. 2008).

\section{A few examples}

We shall limit our choice of examples to two extreme cases: $\beta$ Cephei stars, where a limited number of $\kappa$-driven modes are excited, and solar-like stars, where a forest of acoustic modes are stochastically excited by turbulent motions in the underlying convective envelope.

\section{$6.1 \beta$ Cephei stars}

$\beta$ Cephei stars are massive main sequence stars $(M \sim 8$ $\left.12 M_{\odot}\right)$. Their rather evolved state means that low order mixed modes are to be expected. Some of these stars are hybrid pulsators; in addition to the acoustic $\beta$ Cephei-type modes, they also present some SPB-type long period gmodes. With their usually slow rotation velocity, they are excellent candidates to seismically test the extent of overshooting. The question is at present not answered, although some interesting steps have been covered. In a fitting analysis of HD 129929, Aerts et al. (2003) obtained their best 
Fig. 3 Brunt-Väisälä frequency $N$, hydrogen content $X$, and period spacing $\Delta P$ in three evolutionary states of a main al. 2008) sequence $6 M_{\odot}$ star (Miglio et
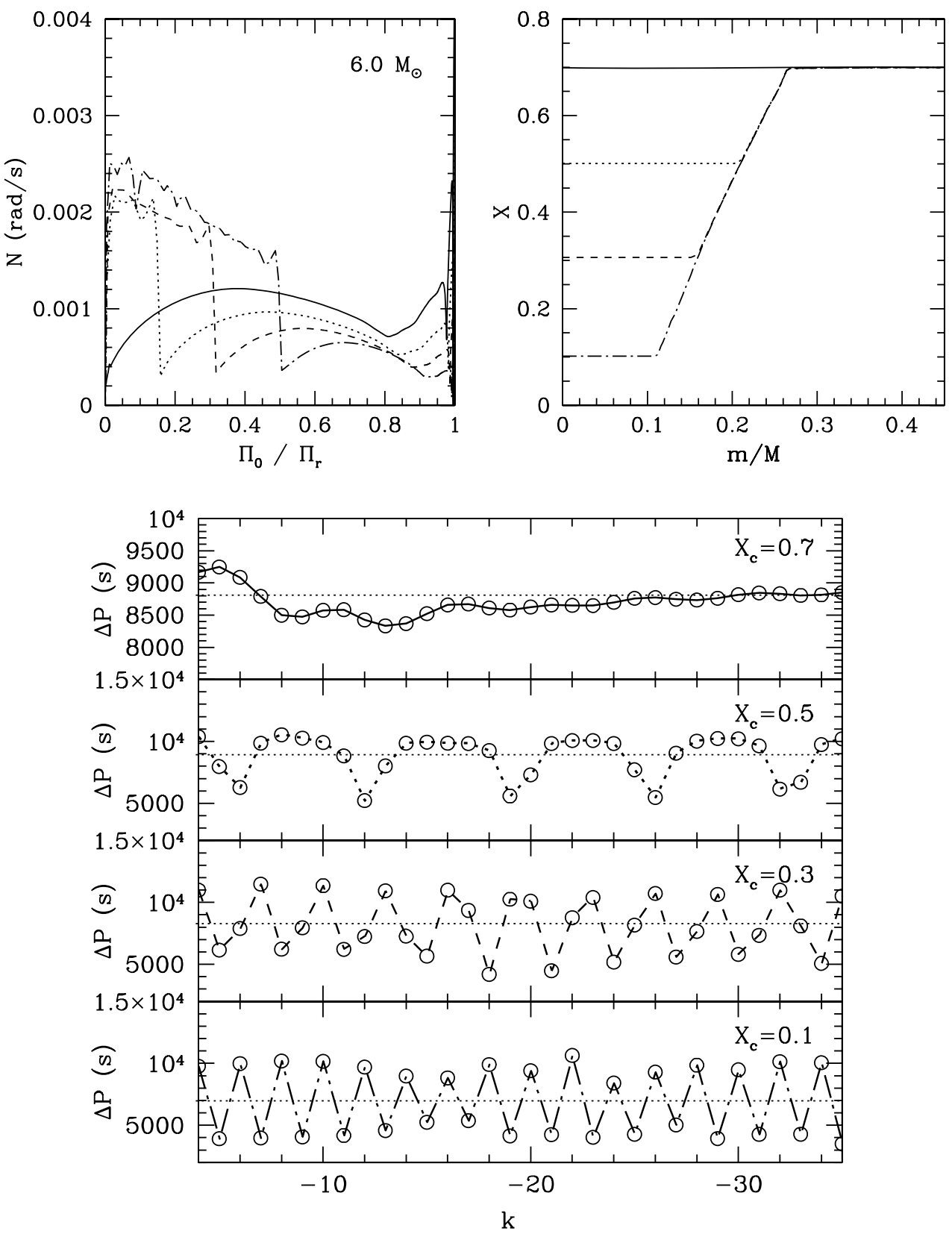

model with a moderate overshooting parameter of the order of 0.1. Through a fitting of five identified modes in $\theta$ Ophiuchi, Briquet et al. (2007) found a much larger overshooting parameter of 0.44 . The situation is less conclusive in other $\beta$ Cephei stars. In 12 Lacertae for instance, Desmet et al. (2009) found an upper limit of about 0.4 for $\alpha_{\text {ov }}$ while Dziembowski and Pamyatnykh (2008) performed a preliminary analysis with models computed without overshooting. The case of $v$ Eridani is even more challenging. Pamyatnykh et al. (2004) advocate a small amount of overshooting $\left(\alpha_{\text {ov }} \leq 0.12\right)$ while Ausseloos et al. (2004) could not find a satisfactory solution based on the fitting of four modes. Although very promising, the status of overshooting in mas- sive stars remains unclear at present. More observations are needed with well-identified modes available, especially in slowly rotating $\beta$ Cephei stars, to help clarify the problem of the extra-mixing in such stars.

\subsection{Solar-like stars}

Low mass stars $\left(M \lesssim 2 M_{\odot}\right)$ have a convective envelope which can stochastically excite acoustic modes. Here the situation is drastically different from that of massive stars since regularities in the frequencies are to be expected due to the asymptotic behavior of the modes. In an analysis of $\eta$ Bootis, Di Mauro et al. (2003) showed how overshooting $\left(\alpha_{\mathrm{ov}}=0.2\right)$ affects the echelle (or folded frequencies with 
Fig. 4 Same as in Fig. 3 for a 1.6 $M_{\odot}$ star computed with overshooting $\left(\alpha_{\mathrm{ov}}=0.2\right.$, thick lines) and without overshooting (thin lines) (Miglio et al. 2008)
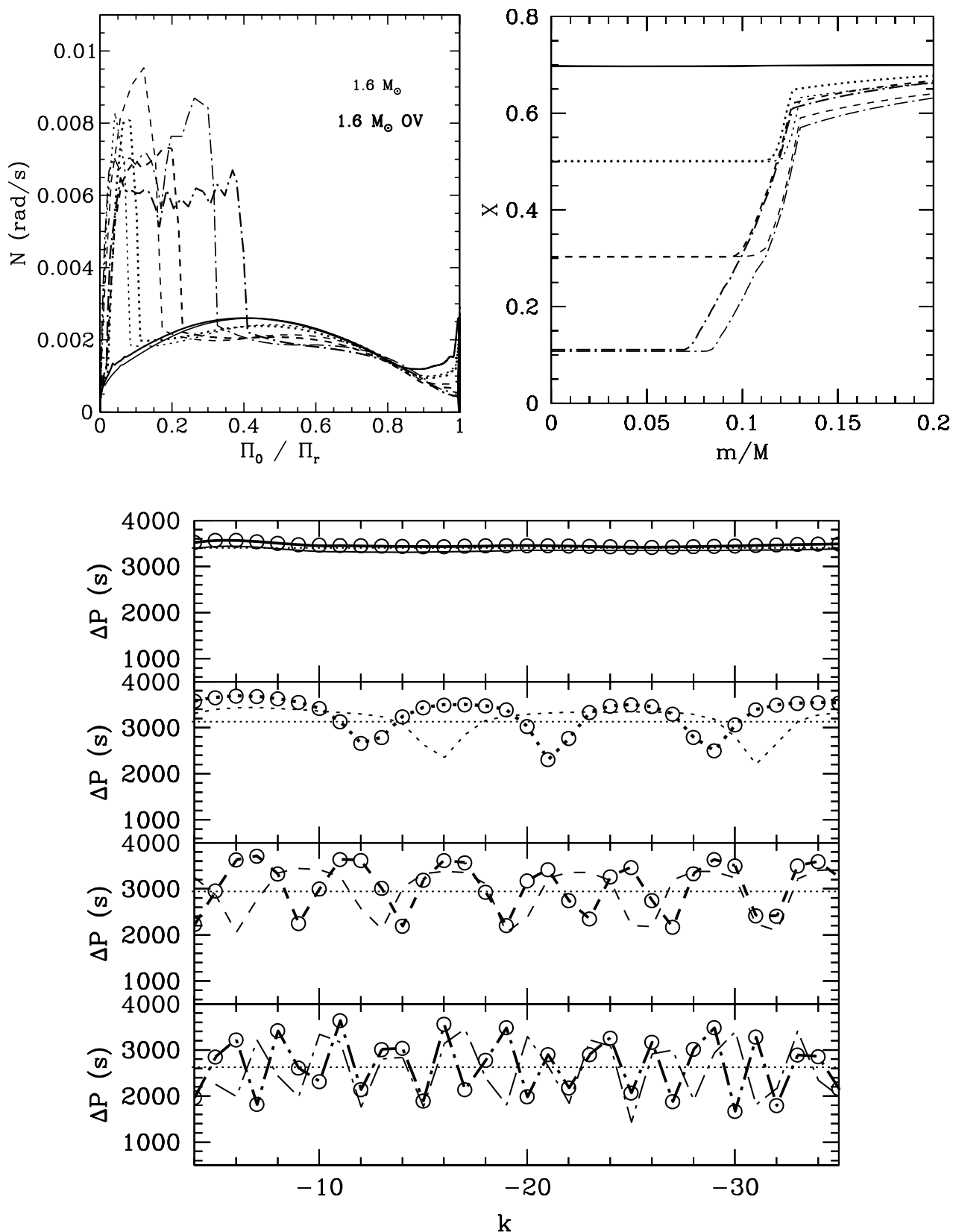

respect to the large separation) diagram, although it was not possible to draw a definitive conclusion in the present status of observations. The CoRoT target HD 49933 poses an interesting challenge. From data collected during the first 60 day run of the CoRoT mission, a beautiful comb-like spectrum typical of solar-like oscillations was obtained for which two equally probable mode identifications have been proposed (Benomar et al. 2009). The best model reproducing the small frequency spacing has an overshooting parameter of 0.7 with one identification and 0.2 with the other. It is hoped that the long run observations of this star (150 days) will discriminate between these identifications (Goupil et al. 2009).
The lack of a precise knowledge of global parameters such as the mass, luminosity, and effective temperature is most often the reason why these analyses cannot reach a clear determination of the amount of overshooting. The best candidates are thus binaries. Miglio et al. (2007) made a theoretical analysis of the asteroseismic properties of the A component (large and small separations, $l=0-1$ ) of 12 Boötis with different assumptions of the amount of overshooting ( $\alpha_{\mathrm{ov}}=0.06$ to 0.037$)$. Although the B component remains a main sequence star in all cases, the A component can be either near the end of the main sequence or already in a post main sequence state with drastic differences in the large and small separations. The binary system $\alpha$ Centauri 

$6.0 M_{\odot}$ star computed with a turbulent diffusion coefficient $D_{\mathrm{T}}=5000 \mathrm{~cm} \mathrm{~s}^{-2}$ (Miglio et al. 2008)
Fig. 5 Same as in Fig. 3 for a
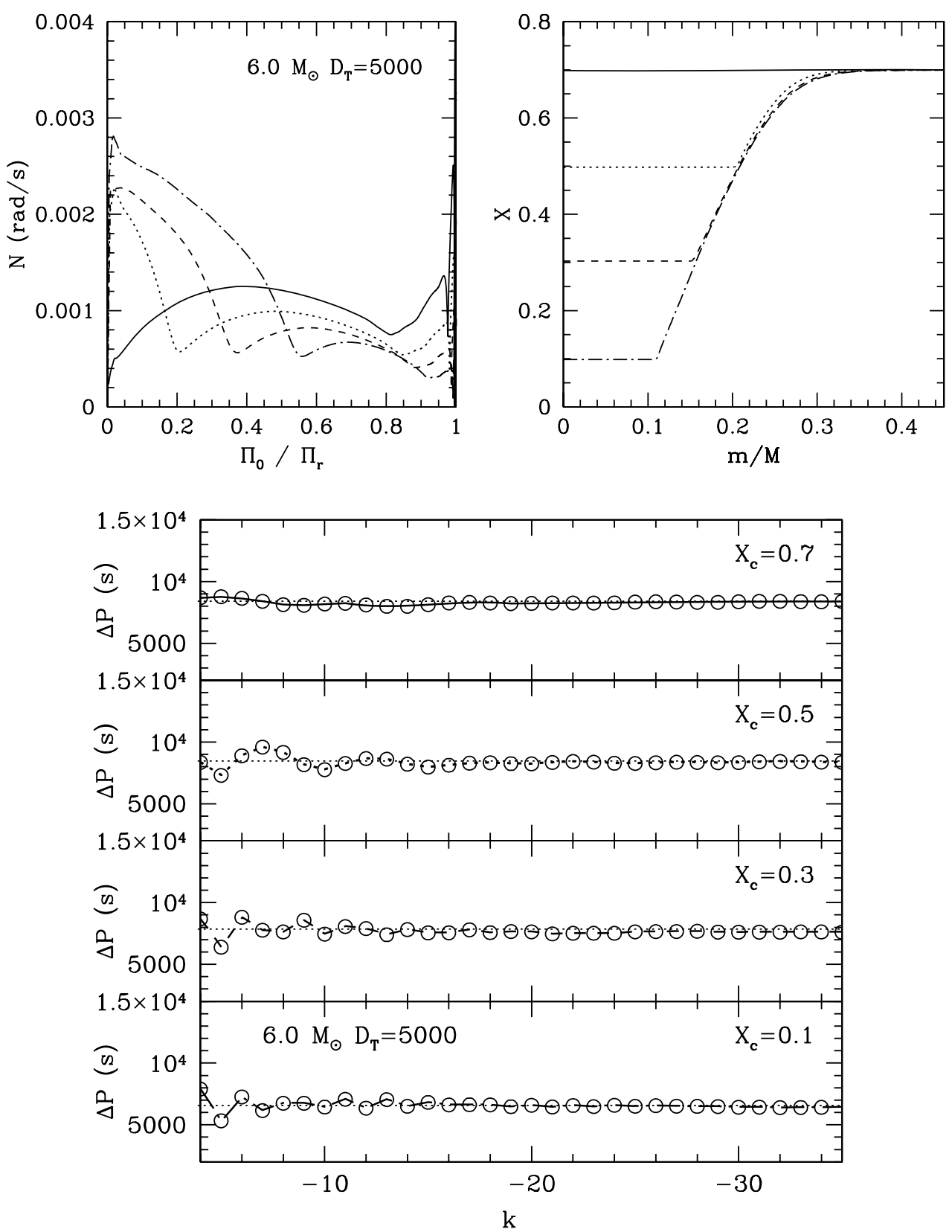

has also been widely seismically investigated (see Miglio and Montalbán 2005 and references therein). Using the freteroseismic data it seems that $\alpha$ Cen A has no convective core.

quency ratios advocated by Roxburgh and Vorontsov (2003) as being the best indicator of the interior structure, Miglio and Montalbán (2005) showed that significant differences in $r_{10}$ were found with $\left(\alpha_{\mathrm{ov}}=0.2\right)$ or without overshooting. The A component keeps a convective core only when overshooting is adopted, and their results pointed towards the absence of such a convective core, although this assertion was not fully conclusive. In a recent analysis, de Meulenaer et al. (2009) combined the data obtained with CORALIE, UVES, and UCLES and were able to set a limit on the overshooting parameter and to ascertain the conclusion: from as-

\section{Conclusions}

The vast majority of main sequence stars have a convective core. The amount of extra-mixing in layers surrounding this core is one major unknown in stellar modeling, whether this mixing comes from overshooting, semiconvection, or turbulence induced by rotation. We have shown that important structural changes are found when such an additional mixing is taken into account. We have also discussed the 


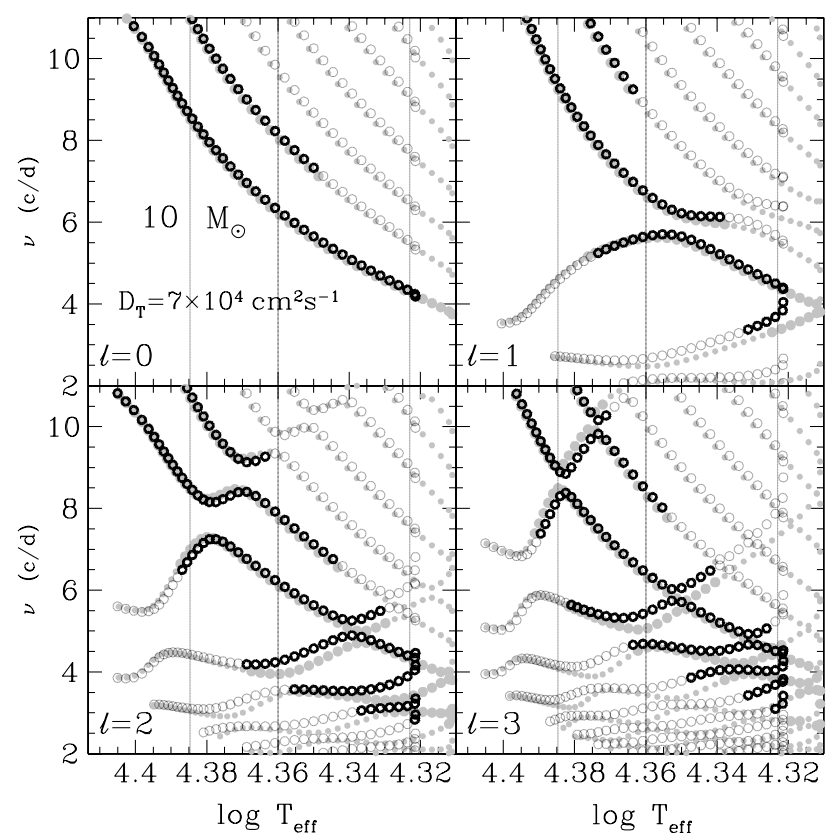

Fig. 6 Frequencies of $l=0-3$ modes as a function of $\log T_{\text {eff }}$ for main sequence models of a $10 M_{\odot}$ star computed with instantaneous overshooting $\left(\alpha_{\mathrm{ov}}=0.1\right.$, gray dots $)$ and a turbulent diffusion coefficient $\left(D_{\mathrm{T}}=7 \times 10^{4} \mathrm{~cm} \mathrm{~s}^{-2}\right.$, open circles $)$. Excited modes are represented by thicker symbols. Vertical lines indicate the location of models with a central hydrogen content of 0.5, 0.3, and 0.1 (left to right) (Montalbán et al. 2008)

possible asteroseismic signatures and their potential in solving this problem. Not only can theoretical asteroseismology discriminate between models with or without extra-mixing but, through the exact shape of the $\mu$-gradient profile, it can shed new light on the true origin of the mixing. This however requires the best possible estimations of the global stellar parameters and the best possible frequency determinations and mode identifications. High precision photometry and spectroscopy together with long duration asteroseimic space- and ground-based observations will reach this goal in a conceivable future.

Acknowledgements JM acknowledges financial support from the Prodex-ESA contract Prodex 8 CoRoT (C90199), and AM is thankful to the Fonds de la Recherche Scientifique (FNRS) for a postdoctoral fellowship. AN and JM are grateful to HELAS for financial support.

\section{References}

Aerts, C., Thoul, A., Daszyńska, J., Scuflaire, R., Waelkens, C., Dupret, M.A., Niemczura, E., Noels, A.: Science 300, 1926 (2003)

Aizenman, M., Smeyers, P., Weigert, A.: Astron. Astrophys. 58, 41 (1977)

Andersen, J., Clausen, J.V., Nordstrom, B.: Astrophys. J. 363, L33 (1990)

Audard, N., Provost, J., Christensen-Dalsgaard, J.: Astron. Astrophys. 297, 427 (1995)

Ausseloos, M., Scuflaire, R., Thoul, A., Aerts, C.: Mon. Not. R. Astron. Soc. 355, 352 (2004)
Benomar, O., Appourchaux, T., Baudin, F., et al..: Astron. Astrophys. 506, 15 (2009)

Bertelli, G., Bressan, A., Chiosi, C., Angerer, K.: Astron. Astrophys. Suppl. Ser. 66, 191 (1986)

Brassard, P., Fontaine, G., Wesemael, F., Hanse, C.J.: Astrophys. J. Suppl. Ser. 80, 369 (1992)

Bressan, A.G., Chiosi, C., Bertelli, G.: Astron. Astrophys. 102, 25 (1981)

Briquet, M., Morel, T., Thoul, A., Scuflaire, R., Miglio, A., Montalbán, J., Dupret, M.A., Aerts, C.: Mon. Not. R. Astron. Soc. 381, 1482 (2007)

Chiosi, C., Maeder, A.: Annu. Rev. Astron. Astrophys. 24, 329 (1986)

Chiosi, C., Bertelli, G., Meylan, G., Ortolani, S.: Astron. Astrophys. 219, 167 (1989)

Christensen-Dalsgaard, J.: Astrophys. Space Sci. 316, 13 (2008)

Claret, A.: Astron. Astrophys. 475, 1019 (2007)

Degl'Innocenti, S., Prada Moroni, P.G., Marconi, M., Ruoppo, A.: Astrophys. Space Sci. 316, 25 (2008)

Demarque, P., Sarajedini, A., Guo, X.-J.: Astrophys. J. 426, 165 (1994)

Demarque, P., Guenther, D.B., Li, L.H., Mazumdar, A., Straka, C.W.: Astrophys. Space Sci. 316, 31 (2008)

de Meulenaer, P., Carrier, F., Miglio, J., Bedding, T., Bouchy, F., Eggenberger, P., Kjeldsen, H., Montalban, J.: Astron. Astrophys. (2009, in preparation)

Desmet, M., Briquet, M., Thoul, A., et al.: Mon. Not. R. Astron. Soc. 396, 1460 (2009)

Di Mauro, M.P., Christensen-Dalsgaard, J., Paterno, L., D’Antona, F.: Sol. Phys. 220, 185 (2003)

Dziembowski, W.A., Pamyatnykh, A.A.: Astron. Astrophys. 248, L11 (1991)

Dziembowski, W.A., Pamyatnykh, A.A.: Mon. Not. R. Astron. Soc. 385, 2061 (2008)

Eggenberger, P., Meynet, G., Maeder, A., Hirschi, R., Charbonnel, C., Talon, S., Ekström, S.: Astrophys. Space Sci. 316, 43 (2008)

Gabriel, M., Noels, A.: Astron. Astrophys. 54, 631 (1977)

Godart, M.: DEA thesis, Liège University, Belgium (2006)

Godart, M.: CoAst 150, 185 (2007)

Goupil, M.J., Michel, E., Lebreton, Y., Baglin, A.: Astron. Astrophys. 268, 546 (1993)

Goupil, M.J., Deheuvels, M.J., Provost, J., et al.: Astron. Astrophys. (2009, submitted)

Grossman, S.A., Taam, R.E.: Mon. Not. R. Astron. Soc. 283, 1165 (1996)

Hui-Bon-Hua, A.: Astrophys. Space Sci. 316, 55 (2008)

Kato, S.: Publ. Astron. Soc. Jpn. 18, 374 (1966)

Langer, N., El Eid, M.F., Fricke, K.J.: Astron. Astrophys. 145, 179 (2008)

Maeder, A., Meynet, G.: Astron. Astrophys. Suppl. Ser. 89, 451 (1991)

Miglio, A., Montalbán, J.: Astron. Astrophys. 441, 615 (2005)

Miglio, A., Montalbán, J., Maceroni, C.: Mon. Not. R. Astron. Soc. 377, 373 (2007)

Miglio, A., Montalbán, J., Noels, A., Eggenberger, P.: Mon. Not. R. Astron. Soc. 386, 1487 (2008)

Miglio, A., Montalbán, J., Eggenberger, P., Noels, A.: CoAst 158, 233 (2009)

Montalbán, J., Miglio, A., Eggenberger, P., Noels, A.: Astron. Nachr. 329, 535 (2008)

Montgomery, M.H., Metcalfe, T.S., Winget, D.E.: Mon. Not. R. Astron. Soc. 344, 657 (2003)

Morel, P., Lebreton, Y.: Astrophys. Space Sci. 316, 61 (2008)

Pamyatnykh, A.A., Handler, G., Dziembowski, W.A.: Mon. Not. R. Astron. Soc. 350, 1022 (2004)

Popielski, B.L., Dziembowski, W.A.: Acta Astron. 55, 177 (2005)

Ribas, I., Jordi, C., Giménez, Á.: Mon. Not. R. Astron. Soc. Lett. 318, L55 (2000)

Roxburgh, I.W.: Astron. Astrophys. 65, 281 (1978) 
Roxburgh, I.W.: Astron. Astrophys. 211, 361 (1989)

Roxburgh, I.W.: Astrophys. Space Sci. 316, 75 (2008)

Roxburgh, I.W., Vorontsov, S.V.: Astron. Astrophys. 411, 215 (2003)

Scuflaire, R., Thédo, S., Montalbán, J., Miglio, A., Bourge, P.O., Godart, M., Thoul, A., Noels, A.: Astrophys. Space Sci. 316, 83 (2008)

Silva-Aguirre, V., Ballot, J., Serenelli, A., Weiss, A.: Astrophys. Space Sci. this colloquium (2009)

Stothers, R.B., Chin, C.-W.: Astrophys. J. 204, 472 (1976)
Stothers, R.B., Chin, C.-W.: Astrophys. J. 390, 136 (1992)

Ventura, P., Zeppieri, A., Mazzitelli, I., D’Antona, F.: Astron. Astrophys. 334, 953 (1998)

Ventura, P., D’Antona, F., Mazzitelli, I.: Astrophys. Space Sci. 316, 93 (2008)

Weiss, A., Schlattl, H.: Astrophys. Space Sci. 316, 99 (2008)

Zahn, J.P.: Astron. Astrophys. 252, 179 (1991) 